\title{
Fundamentos para el estudio comparativo de las formas de construcción de la violencia de género como problema público- político en la historia reciente de México y Argentina.
}

Pedro Cerruti

Doctor en Ciencias Sociales (Universidad de Buenos Aires) Docente - Investigador en la Universidad de Buenos Aires Investigador en la Universidad Nacional de Quilmes, pedrocerruti@gmail.com

\begin{abstract}
Resumen
La violencia de género constituye un problema global de particular incidencia en la región latinoamericana, razón por la cual ha sido reconocida como una cuestión que atañe a la salud, que constituye un obstáculo para el desarrollo y un grave problema de Derechos Humanos. El presente trabajo da cuenta de los aspectos fundamentales para el estudio comparativo de sus formas de construcción como problema público-político en la historia reciente de México y Argentina. Además, se especifican los avances en cuanto a la indagación comparativa de los procesos socio-históricos que, en ambos países con sus particularidades, permiten establecer las formas de emergencia de la problemática en la esfera pública de la mano del activismo feminista y la aparición de organizaciones civiles; y los modos en que la temática se incorpora en la agendas legislativas y de gobierno, en el contexto de su tematización por organismos internacionales.
\end{abstract}

Palabras clave: violencia de género; Latinoamérica; Argentina; México; historia reciente

\section{Introducción}

$\mathrm{L}$ A VIOLENCIA DE GÉNERO, específicamente la violencia contra la mujer ${ }^{1}$, constituye una de las formas de violencia que -más allá de sus variaciones en función de los contextos culturales en los que se ejerce- posee una presencia universal y por ello configura un problema global (Heise et al., 1999). Constituye un fenómeno de particular gravedad, en primer lugar, por ser una de las formas más frecuentes, persistentes y extendidas de violencia y de particular incidencia en Latinoamérica, al mismo tiempo que parece continuar en aumento (inclusive en contextos de disminución de otras formas de violencia) (Guerrero, 2002; López Pons, 2010).

1 Existen variadas formas de denominar el fenómeno en cuestión que expresan diversos enfoques de la temática, tales como "violencia sexista" (Gutiérrez Castañeda, 2004), "violencia por razón de sexo" (RAE, 2004), "violencia machista" (García, 2009). Para los fines del presente artículo, la violencia contra las mujeres es entendida y denominada desde una perspectiva de género que será precisada oportunamente. 
En segundo lugar, por la variedad de sus formas -fisica, psicológica, sexual, etc.- y sus manifestaciones, que incluyen la violencia familiar, sexual, el feminicidio, la trata, etc. (Rico, 1992; Brendel, 2003; Contreras et al., 2010; Jiménez e Roderos, 2010); y por las cualidades de sus modos de ejercicio que incluyen su carácter frecuentemente letal y la utilización de medios de una brutalidad y crueldad particular (Incháustegui e de la Paz, 2011).

En tercer lugar, por constituir una forma extrema de discriminación y dominación de género que, por lo tanto, es el emergente de las relaciones de poder y de la desigualdad entre el varón y la mujer que todavía atraviesan a las sociedades contemporáneas, que aún hoy presentan un alto grado de tolerancia, y porque ha sido objeto de una invisibilización histórica (Milosavljevic, 2007). Y, por último, por el modo en que en América Latina constituye un fenómeno que ve agravadas sus manifestaciones y consecuencias por contextos de pobreza, desigualdad y exclusión social (AI, 2009), así como de impunidad y de dificultades particulares de acceso a la Justicia (OEA/CIDH, 2007).

Por todo ello, la violencia contra la mujer ha sido considerada como una cuestión que atañe a la salud de las poblaciones (Velzeboer, 2003) y constituye un obstáculo para el desarrollo de la región (Carrillo e Bunch, 1991), así como un grave problema de Derechos $\mathrm{Hu}-$ manos (Rico, 1996).

El presente artículo busca presentar los fundamentos y avances de un proceso de investigación en curso que, tomando en cuenta las consideraciones antedichas, se ha propuesto indagar en los factores socio-políticos y culturales que en la historia reciente de las sociedades argentina y mexicana han incidido en los modos de problematización social de la violencia de género $y$ en las características que han adquirido las respuestas sociales frente a la misma. Para ello, se ha propuesto comparar los discursos hegemónicos sobre la violencia de género en Argentina y México, teniendo en cuenta sus contextos de producción y circulación e identificando las concomitancias y las divergencias entre ambos, para reconstruir las matrices discursivas dominantes a través de las cuales se conforma la violencia de género como problema público-político en los debates de la agenda pública de ambos casos de estudio. El objetivo, en última instancia, es el de poder producir una genealogía que presente en una visión de conjunto los procesos y acontecimientos que produjeron transformaciones en las matrices discursivas sobre la violencia de género en los dos países, queriendo en el horizonte contribuir a la comprensión de las características del fenómeno en Latinoamérica, una región especialmente afectada por él, pero que al mismo tiempo ha sido el lugar de importantes y tempranos procesos de visibilización y reconocimiento del tema como un problema de Derechos Humanos.

\section{La violencia de género como problema público-político}

Ahora bien, una de las problemáticas que ha sido destacada respecto de la temática en cuestión, y que es particularmente pertinente para el caso de América Latina, es el hecho de que a pesar de que desde hace por lo menos dos décadas el tema ha sido incorporado en la agenda internacional en el ámbito de los Derechos Humanos -dando lugar a su reconocimiento, a la adopción de instrumentos específicos para contrarrestarla, a la adopción de legislaciones y políticas sociales nacionales destinadas al tratamiento y protección de las víctimas, a la creación de organismos de toda escala dedicados a la temática, etc. (OPS/OMS, 2005)constituye un fenómeno persistente y que inclusive parece agravarse (Fríes e Hurtado, 2010).

Eso ha generado múltiples interrogantes que abarcan un amplio espectro de problemáticas, entre las cuales se han destacado la eficacia real de las formas en que se han diseñado e implementado los marcos jurídicos y las políticas públicas (Sagot, 2008); las dificultades vinculadas con la producción y análisis de la información en materia de evaluación, monitoreo e impacto de las mismas (Almerás e Calderón Magaña, 2012); y las características específicas de los modos en que la problemática ha sido visibilizada, conceptualizada e introducida en la agenda pública.

Este último interrogante involucra la dimensión propiamente cultural del fenómeno, que abarca los aspectos comunicacionales y las aristas relativas a las formas de la discursividad social que participan de su conformación. El Encuentro Mundial "La Comunicación como fuente de poder para las Mujeres", realizado en Bangkok en 1994 (Bhasin, 1994), y el Simposio Internacional sobre Mujeres y Medios de Comunicación organizado por la UNESCO en Toronto en 1995 (UNESCO, 1995) constituyen hitos en el reconocimiento de esta dimensión como una problemática con estatuto propio en la agenda internacional.

Se trata de una cuestión que presenta una complejidad particular ya que en ella, por un lado, participan múltiples actores tanto pertenecientes al sistema político como a la sociedad civil, lo cual incluye desde organizaciones no gubernamentales hasta víctimas y sus familiares, y desempeñan un rol destacado los medios masivos de comunicación; y por otro, involucra desde campañas programáticas de sensibilización y difusión de derechos hasta formas múltiples, inorgánicas y discordantes de producción y circulación de discursos públicos -noticias periodísticas, productos de la industria cultural, relatos testimoniales, difusión de estadísticas y estudios de diversa índole, etc.- que tratan de manera directa o indirecta el problema.

En este sentido, la temática ha transitado un recorrido en la historia reciente que la ha llevado de considerarse 
una cuestión "privada" a transformarse en una problemática "pública" (Bolles et al., 2006). Uno de los principales focos de atención ha sido su tratamiento en los discursos periodísticos (Rodigou, 2007); sin embargo, la cuestión involucra de manera general a los diferentes formatos (gráfica, televisión, etc.) y géneros (periodísticos, ficcionales, de "telerealidad", publicitarios, etc.) de la comunicación masiva a través de los cuales se ha producido su visibilización social. La importancia de esta dimensión comunicacional radica en el hecho de constituir un espacio privilegiado de intervención con miras a la sensibilización y lucha contra la violencia como de reproducción de las matrices culturales que la legitiman y favorecen (Poppe, 1997), lo cual ha llevado a la reflexión sobre el problema y a la propuesta de criterios éticos y estratégicos para la producción de información relativa a la violencia contra las mujeres (Drezin, 2001) y a la conformación de observatorios regionales dedicados a su seguimiento (Morelli e Rey, 2012).

La importancia de la temática, la naturaleza de los interrogantes planteados respecto de sus formas de visibilización, las características de los contextos socio-políticos e histórico-culturales en los que se ha desenvuelto su problematización y la necesidad de abordar la cuestión en su doble dimensión global y local, conducen a reconocer la necesidad de interrogarse respecto de las maneras en que se ha producido la construcción de la violencia contra la mujer como problema públicopolítico en diferentes países de la región latinoamericana.

Dicho problema puede ser desagregado a su vez en una serie de interrogantes ya que implica preguntarse, en principio, cuáles son las características de las formas de inclusión de la temática en las agendas de las organizaciones de la sociedad civil, de los medios masivos de comunicación y de las instancias legislativas y de gobierno; y qué transformaciones se han producido en las formas de visibilización de la temática a lo largo del tiempo. Al mismo tiempo, requiere examinar qué diferencias y qué continuidades existen entre los modos en que se han desenvuelto esos procesos en diferentes países de la región, tales como Argentina y México; y qué relación se puede establecer entre esas formas de problematización y los contextos históricos, culturales y políticos nacionales específicos, por un lado y, por otro, las tendencias regionales e internacionales. Por último, requiere examinar qué implicancias pueden extraerse de esas formas de construcción de la temática desde el punto vista de la promoción de la erradicación de la violencia contra la mujer y de la igualdad de género en la región en el futuro.

Para responder dichos interrogantes, se hace necesario un abordaje socio-cultural de la construcción de los problemas sociales en la esfera público-política. Ello implica, en lo que se refiere al modelo de análisis e interpretación, la perspectiva de una sociología interpretativa de la dimensión cultural de los fenómenos sociales (Margulis, 2009). Desde este punto de vista, se entiende a los fenómenos sociales como procesos de significación y de producción de sentido, sujetos a su vez a condiciones que forman parte del horizonte cultural -esto es, las significaciones compartidas por medio de las cuales un colectivo se construye una representación de sí mismo y de su realidad social-del momento histórico en el que se desarrollan; y se considera que es en el nivel de la discursividad en el que esa doble dimensión (producción de sentido y condicionamiento socio-cultural) se materializa de manera privilegiada y se vuelve analizable (Verón, 2004).

Dicho modelo se focaliza, por su parte, en función del estudio de problemas sociales entendiéndolos como "constructos sociales" (Loseke e Best, 2003), es decir, siguiendo una perspectiva construccionista de la realidad social en función de la cual se postula que la emergencia de los problemas sociales, su definición y conceptualización, su legitimación y su capacidad para movilizar acciones, es el resultado de procesos históricos que involucran eventos socio-políticos, disputas y relaciones de poder entre grupos sociales y conformaciones de consensos y hegemonías.

El ámbito privilegiado de desenvolvimiento de dichos procesos sociales es el "espacio público-político", entendiéndolo como una esfera de comunicación (Habermas, 1999) en la cual participan tanto actores de la sociedad civil como del sistema propiamente político y determinados problemas son visibilizados, identificados y definidos, en el sentido de ser interpretados y tematizados, poniendo en juego estrategias de elaboración de la información dependientes de las relaciones de poder que determinan oportunidades diferenciales de ejercer influencia en las disputas por la imposición de sentidos (Habermas, 2001); en otras palabras, en ella se desenvuelven los procesos de producción de las matrices discursivas que componen la construcción de la realidad social y, por lo tanto, que nos brindan acceso a ella.

Esta modalidad de abordaje involucra una perspectiva de análisis del discurso que lo considera desde el punto de vista de su "performatividad" (Austin, 2003), es decir, su capacidad para hacer existir los objetos de los que se habla, construirlos de maneras específicas y atribuirles determinados criterios de veridicción. Esa capacidad productiva de los discursos depende de sus disposiciones en ciertos regímenes de saber y relaciones de poder (Foucault, 2008) y de las posiciones de enunciación de los diferentes actores sociales en el seno de las relaciones de fuerza que configuran las disputas por la construcción de formaciones discursivas hegemónicas (Laclau e Mouffe, 1987). Este último aspecto implica reconocer la relevancia particular que poseen en las sociedades contemporáneas los medios masivos de comunicación en general, y determinados medios hegemónicos en particular, en tanto que intervienen en calidad de actores sociales y políticos en el escenario público-político (Borrat, 1989) al mismo tiempo que poseen un poder estructurante del mismo por su 
capacidad para la regulación de la producción y circulación de los discursos y por ello para la construcción de las agendas públicas (McCombs, 2006).

En ese sentido, en lo que se refiere a la selección de fuentes documentales, se consideran tanto documentos producidos por las instituciones legislativas y de gobierno tales como materiales vinculados a disposiciones de creación de organismos y programas; estatutos de las agencias dedicadas al problema en sus diferentes niveles; campañas de difusión; legislaciones, etc.; como documentos producidos por organizaciones civiles dedicadas a la temática con una trayectoria y relevancia reconocida y una permanencia en el tiempo que permita abarcar el período de análisis en su totalidad. Junto con ello, es fundamental el análisis de material proveniente de fuentes hemerográficas que incluya aquellos diarios de circulación nacional más importantes en términos de tirada y de relevancia en la opinión pública y que permitan circunscribir un amplio margen del espectro político ${ }^{2}$. A su vez, debe ser destacada la importancia de los documentos de índole jurídica, por su papel en la institución de órdenes de normatividad y de saberes y criterios de veridicción, lo cual implica analizarlos en función del modo en que producen efectos performativos de puesta en forma de objetos sociales (Foucault, 2007).

Ahora bien, la dimensión histórica involucrada en el desarrollo de los casos de estudio a lo largo del período de tiempo seleccionado es analizada desde la perspectiva de la historia del presente (Díaz Barrado, 1998). Se considera que dicho enfoque es necesario en tanto los procesos indagados continúan vivos, en constante devenir y en un desarrollo inconcluso, y que esa dimensión actual del fenómeno no solo comporta una relación de coetaneidad con la investigación aquí proyectada sino que sigue constituyendo una urgencia y un problema prioritario para las sociedades del presente. En ese sentido, se considera que el período de análisis seleccionado constituyó un momento de profunda transformación con decisivos efectos en la morfología de las sociedades mexicana y argentina contemporáneas. Justamente, la historia del presente constituye una perspectiva de análisis crítica que involucra reincorporar los procesos actuales en su dimensión histórica, esto es considerando sus transformaciones a lo largo del tiempo, como paso ineludible para comprender su presente y extraer conclusiones de cara al futuro.

En este contexto, la genealogía (Dean, 1994), ha sido considerada como un procedimiento interpretativo en función del cual se buscan rastrear los procesos y acontecimientos que generaron puntos de inflexión, en tanto que provocaron transformaciones y emergencias de singularidades en los elementos que constituyeron el problema en cuestión, y comprender los efectos que produjeron en su devenir y en su modo de existencia en el presente.

Además, desde esta modalidad de análisis se incorpora una perspectiva que considera al género como un conjunto complejo de relaciones y procesos que se encuentran en vinculación con otras formas de relaciones socioculturales pero que poseen un estatuto propio en la conformación histórica de los problemas planteados y cuyas implicancias deben ser reconocidas. En otras palabras, la dimensión de género tiene efectos directos en los procesos de producción de sentido, en la estructuración de las redes de poder que atraviesan todo el entramado social y de las dinámicas comunicacionales que organizan las disputas en la esfera de la opinión pública (Lamas, 2000; Gamba, 2007). Por lo tanto, los modos de construcción de identidades de género establecen las condiciones de desigualdad entre varones y mujeres que estructuran el campo de emergencia de la violencia que es objeto de estudio (Segato, 2010).

\section{Argentina y México: aspectos fundamentales de su estudio comparativo}

Como se mencionó anteriormente, la investigación se ha centrado en el análisis comparativo del desarrollo de casos. La elección de un estudio comparado (Mahoney e Rueschemeyer, 2003) de esta naturaleza obedece a que, como ha sido destacado, por un lado, la violencia de género constituye un fenómeno global que, si bien adquiere formas particulares en función de los diferentes contextos culturales, ha sido reconocido como un problema que afecta a todas las sociedades contemporáneas; y, por otro, a que sus formas de problematización en la historia reciente ponen en contacto los planos global, regional y nacional según las dinámicas de los intercambios y los procesos de difusión transcultural que caracterizan al mundo globalizado. En un contexto como este, la comparación constituye un método necesario para producir explicaciones comprensivas de los fenómenos y procesos específicos de cada caso, a partir del reconocimiento de aquellos factores relevantes que forman parte de un horizonte regional y/o global. En otras palabras, un análisis comparado es necesario, por un lado, para abordar el objeto de estudio en la doble dimensión local o nacional y global o regional que le es inherente; $y$, por otro, para comprender las particularidades histórico-culturales y las especificidades socio-políticas que le dan al objeto de estudio su 
singularidad al interior de un país o un Estado-Nación en relación con un horizonte de regularidades que configura una dinámica transnacional.

Para estos fines, se eligieron los casos de Argentina y México, y dentro de ambos países se circunscribirá el análisis específicamente a la Ciudad de México y de Buenos Aires. Esta última delimitación responde al estatuto que posee lo urbano en la conformación del problema de estudio. Como parte de un fenómeno global de particular incidencia en la región latinoamericana, en ambos países se ha producido en las últimas décadas una tendencia a la consolidación de la vida urbana y un aumento de la población residente en las grandes ciudades en relación con la población rural (de Mattos, 2010). Ejemplos paradigmáticos de ello son las capitales de ambos países, las cuales constituyen dos de las tres ciudades más pobladas y extensas de la región, si bien existen diferencias significativas entre ambas que hacen que la Ciudad de México se considere, además, una de las megalópolis más importantes del mundo En ese proceso, la centralidad de la ciudad ha crecido exponencialmente en tanto espacio de los principales procesos de transformación económica, política y social y como terreno experiencial clave en la producción de las formas culturales hegemónicas, en el ejercicio de la ciudadanía y en la construcción de lo público, al mismo tiempo que es el lugar privilegiado de territorialización de las esferas de intercambio simbólico producidas por los medios masivos de comunicación (Martín Barbero, 2008). Al mismo tiempo que constituyen los espacios en lo que se intersectan los procesos globales con las realidades locales, conviven allí, en un incrementado nivel de hacinamiento y en permanente tensión, la modernización y el atraso, y la integración y la exclusión social, produciendo geografias fragmentadas por los procesos de desigualdad y segregación social y económica (Portes et al., 2008). Justamente son estos escenarios urbanos los que han constituido el soporte físico y cultural en el cual en la historia reciente se ha producido la emergencia de la cuestión de la violencia en general, y de la violencia de género en particular, como tema de la agenda pública y es el sitio paradigmático donde se experimentan esas formas de la violencia que allí se problematizan (Falú, 2009). El reconocimiento de esta relación entre el "hábitat" -en tanto producto y soporte de los procesos sociales- y las relaciones de género -cuyas disposiciones asimétricas se expresan en el territorio al mismo tiempo que este participa de su reproducciónse ha visto reflejado, por ejemplo, en la creación en 1989 de la Red Mujer y Hábitat de América Latina (Falú e Rainero, 1995); y, en lo que refiere específicamente al problema de la violencia de género en el hábitat urbano, en el lanzamiento del Programa Regional "Ciudades sin violencia hacia las mujeres, ciudades seguras para todos y todas", por UNIFEM.

\section{Hacia una genealogía de las formas de problematización de la violencia de género}

En dicho período de análisis se ha conseguido delimitar una serie de hitos y momentos significativos en lo que respecta a la genealogía de la construcción del problema que constituye el objeto de estudio, que muestran el modo en que se entrecruzan las dinámicas internas de las sociedades en cuestión con las transformaciones a nivel regional y global impulsadas por el movimiento feminista y los organismos supranacionales. En primer lugar, un primer período que abarca los procesos y acontecimientos que conducen a la formulación de las leyes de primera generación relativas al tratamiento de la violencia familiar o doméstica, la cual pasa de ser considerada un tema privado a visibilizarse y tematizarse en el ámbito público; así como las reformas vinculadas con la legislación penal de los delitos sexuales, las cuales mantenían hasta ese momento una formulación claramente discriminatoria. Todos estos procesos se desenvuelven en un primer momento de emergencia de la problemática en la esfera pública de la mano del activismo feminista y la aparición de organizaciones civiles dedicadas a la temática; y un segundo momento en el que la temática se incorpora en las agendas legislativas y de gobierno, en el contexto de su tematización por organismos internacionales.

Estos momentos coinciden grosso modo con las décadas de los ochenta y los noventa, y con transformaciones socio-políticas y culturales características en cada uno de los países. En el caso de la Argentina corresponde, por un lado, con la recuperación democrática luego de la última dictadura cívico-militar argentina (1983) $y$, por otro, con el estallido de la crisis de 2001 que en nuestro país puso fin al período dominado por el proyecto de reforma neoliberal del Estado, iniciado bajo la presidencia de Carlos Menem (1989-1999) (Bonnet, 2007). En el caso de México, ese período circunscribe las presidencias de Miguel de la Madrid (1982-1988), Carlos Salinas de Gortari (1988-1994) y Ernesto Zedillo (1994-2000). En ese caso, se trata también de un período de reformas neoliberales iniciadas por de la $\mathrm{Ma}-$ drid y profundizadas por Salinas de Gortari, y coincide con un período de democratización que conducirá al fin del período de gobierno continuado del PRI en el año 2000 (Cadena Vargas, 2005). Además, en ambos países, conforme al proceso de democratización y de reforma de las instituciones del Estado a mediados de la década de los noventa, se produce una transformación en lo que respecta a la autonomía política y administrativa de las ciudades capitales las cuales pasan a tener gobiernos elegidos popularmente y, en ambos casos, por fuerzas opositoras a los gobiernos nacionales y de una orientación política de centro izquierda o progresista, 
como es el caso del gobierno de la ALIANZA en la Ciudad de Buenos Aires, a partir de 1996 y del Partido de la Revolución Democrática (PRD) en la Ciudad de México, desde 1997 (Serrano Salazar, 2001; Rabey e Martínez, 2006).

Se trata también de un período de profunda transformación en las agendas de los organismos internacionales y regionales relativas a la temática, en la cual el activismo feminista desempeñó un rol protagónico. Cabe recordar que es en la Ciudad de México donde se realiza la Primera Conferencia Mundial de las Mujeres en 1975, la cual inaugura el Decenio de las Naciones Unidas para la Mujer 1975-1985, para lo cual se crea en 1976 el Fondo de Contribuciones Voluntarias que en 1984 se transformaría en el Fondo de Desarrollo de las Naciones Unidas para la Mujer (UNIFEM); y que es en ese contexto que se establece en 1979 la Convención sobre la eliminación de todas las formas de discriminación contra la mujer (CEDAW), recuperando el espíritu de la Declaración sobre la Eliminación de la Discriminación Contra la Mujer de las Naciones Unidas de 1967 (UN, 2010), pero que no incluye una referencia explícita al tema de la violencia, el cual será incorporado recién durante la Segunda Conferencia Mundial sobre la Mujer, en 1980, bajo la forma de una resolución específicamente referida a "la mujer maltratada y a la violencia en la familia".

Por su parte, el movimiento feminista internacional ya había planteado el tema en otros términos con la organización del Primer Tribunal de Crímenes contra las Mujeres en Bruselas en 1976 (Russell e Van de Ven, 1990). Allí, Diane Russell -quien ya había publicado el pionero Politics of rape (Russell, 1975)-, utiliza por primera vez con un significado político el término femicide al que daría forma acabada años más tarde en su participación en el trabajo colectivo Femicide. The politics of women killing (Radford e Russell, 1992). En nuestra región, el activismo feminista latinoamericano, ya en 1981, durante el I Encuentro Feminista Latinoamericano y del Caribe, se manifiesta de modo explícito sobre el problema al resolver la necesidad de exigir a los organismos internacionales la investigación de los casos de violación a los Derechos Humanos de las mujeres, y establecer el 25 de noviembre como el Día Internacional No Más Violencia Contra las Mujeres, que luego sería reconocido por la ONU en 1999 (Restrepo e Bustamante, 2009). La elección de dicha fecha, en memoria del asesinato, en la República Dominicana, de las hermanas Mirabal en 1960 a causa de su oposición al régimen dictatorial de Rafael Trujillo, es significativa respecto del modo en que se ha producido el reconocimiento de la problemática en la región, ya que muestra cómo esta se inscribe en la estela abierta por las formas de resistencia contra los regímenes autoritarios latinoamericanos y la memoria de la victimización a manos del Estado en tanto grave violación a los Derechos Humanos (Navarro, 1982).

Será en la década de los noventa, a partir de la Conferencia Mundial sobre Derechos Humanos de Viena, en 1993, que el tema se incorporó definitivamente en la agenda de los organismos internacionales en el ámbito de los Derechos Humanos. Para ello fue determinante la Campaña Global por los Derechos Humanos de las Mujeres impulsada especialmente por el Centro para el Liderazgo Global de las Mujeres (CWGL) en la cual, con miras a dicha Conferencia, se organizaron iniciativas orientadas en esa dirección como el establecimiento en 1991 de la Campaña 16 días de Activismo contra la Violencia de Género, que se sigue realizando anualmente hasta la fecha, así como el Primer Tribunal Mundial de Violaciones a los Derechos Humanos de las Mujeres cuyo trabajo se desarrolló durante los años 1992 y 1993 (Rico, 1996). Para 1992, se había incorporado a la CEDAW la Recomendación General Nro. 19 referida específicamente a la violencia contra la mujer y que incorpora a su vez temas como el hostigamiento sexual y la explotación. Así, en 1993, con estos antecedentes, la Asamblea General de las Naciones Unidas aprueba la Declaración sobre la eliminación de la violencia contra la mujer en la que se la reconoce como un grave atentado contra los derechos humanos. Este documento es considerado un hito en el modo en que a partir de ese momento la violencia contra la mujer se recorta y tematiza como un problema con un estatuto propio y como un objetivo fundamental a ser tratado por la comunidad internacional (Guerrero, 2002; UN, 2006).

Sin embargo, a nivel de los organismos regionales, en 1990 la Comisión Interamericana de Mujeres de la Organización de Estados Americanos llevaba adelante la Primera Consulta Interamericana sobre la Mujer y la Violencia y adoptaba la Declaración sobre la Erradicación de la Violencia contra la Mujer, que luego daría lugar a la aprobación por parte de la Asamblea General de la OEA de la Convención Interamericana para Prevenir, Sancionar y Erradicar la Violencia contra la Mujer, también llamada Convención de Belém Do Pará, en 1994, haciendo de América Latina la única región que cuenta con un documento específico dedicado a la temática de esa naturaleza (OEA, 1994; Guerrero, 2002).

Específicamente en México, el Movimiento de Liberación de la Mujer (MLM), creado en 1974, y la conformación de la Coalición de Mujeres Feministas en 1976, integrada por diferentes organizaciones, habían ya asumido la denuncia de la problemática de la violencia contra la mujer y planteado la necesidad de una abordaje jurídico específico y del establecimiento de programas de atención dedicados especialmente al tratamiento y apoyo a las mujeres violadas y víctimas de maltrato conyugal. En 1979, esas organizaciones conjuntamente con partidos políticos de izquierda crearon el Frente Nacional Pro Liberación y Derechos de la Mujer (FNALIDM) que recogía, entre otras, a esas reivindicaciones. Un año antes, en 1978, el MLM funda en la Ciudad de México el pionero Centro de Apoyo a Mujeres Violadas (CAMVAC) con el objetivo no solamente de brindar ayuda a las mujeres víctimas 
de violación en los aspectos médicos, psicológicos y jurídicos, sino también de denunciar y crear conciencia respecto de la problemática en la sociedad con el fin de su erradicación. Con este último objetivo publican en 1983 el también pionero "Documento de denuncia y análisis de violación sexual a las mujeres", que coincide con un momento de institucionalización de la temática a través de la creación de diversas organizaciones, como por ejemplo la Asociación Mexicana Contra la Violencia a las Mujeres (COVAC) en 1984, y que da lugar a un proceso que conduce a la conformación de la Red Nacional contra la Violencia hacia las Mujeres en 1989, integrada por agrupaciones de todo el país (Bartra, 1992; Lang, 2003; Díaz Pérez, 2009).

Es en este momento que aparecen, por ejemplo, las organizaciones Centro de Apoyo a Mujeres Violadas (CAMVAC), activo de 1978 a 1989; la Asociación Mexicana Contra la Violencia a las Mujeres A.C. (COVAC), fundada en 1984 y activa hasta el año 2004; el Centro de Apoyo a la Mujer Margarita Magón, A.C, (CAM), fundado en 1986; y el Centro de Investigación y Lucha contra la Violencia Doméstica A. C. (CECOVID) fundada en 1987. En ese mismo año, el diario La Jornada comienza a editar un suplemento dedicado específicamente a temas de género ("Doble Jornada" y luego "Triple Jornada") que se mantendrá hasta 2006, un fenómeno que no sería replicado con esas características en la Argentina sino hasta la publicación de "Las 12" por el diario Página 12 en 1998. Cabe recordar, además, que es en la Ciudad de México que se funda en 1976 la revista Fem, una publicación feminista pionera en América Latina y que continúa editándose hasta el día de hoy.

En la Argentina, por su parte, la recuperación democrática de 1983 produce una renovación del activismo feminista que incluye la introducción del tema de la violencia contra la mujer en la agenda pública bajo el marco de los Derechos Humanos (Maffia, 2002; Gil Lozano, 2004). En un momento de emergencia de organizaciones civiles dedicadas específicamente a la temática o que la incluyen entre sus prioridades, algunos de los hitos que se han destacado en ese proceso son la organización del Tribunal de Violencia contra la Mujer en 1983 (Chejter, 1995); la creación de la Multisectorial de la Mujer, con participación de integrantes de organizaciones sociales, feministas, partidos políticos y sindicatos, y la organización de manifestaciones públicas en las cuales la violencia contra la mujer era una cuestión central, tales como las multitudinarias marchas a Plaza de Mayo con la consigna "Violación es tortura"; la creación en la Asamblea Permanente por los Derechos Humanos de un área específica dedicada a los derechos de la mujer en 1987, con la incorporación de la problemática de la violencia; y la conformación de la Red Nacional contra la Violencia Doméstica en 1988, con la participación de organizaciones de todo el país; entre otros (CECYM, 1996; Barrancos, 2012).
En este contexto aparecen, por mencionar algunas, las organizaciones Asociación Trabajo y Estudio de la Mujer (ATEM), activa desde 1982; Lugar de mujer, fundada en 1983; la Asociación Argentina de Prevención de laViolencia Familiar (AAPVF), creada en 1985; el Movimiento Feminista-Tribunal deViolencia contra la Mujer, establecido en 1984; y la Fundación para el Estudio e Investigación de la Mujer (FEIM), creada en 1989. Además, surgen en ese momento el suplemento "La Mujer" publicado en el periódico Tiempo Argentino entre 1982 y 1986; el periódico Alfonsina, dirigido por María Moreno, la primera publicación feminista de la posdictadura en circulación entre diciembre de 1983 y julio de 1984; y la página Mujer de Sur publicada entre 1989 y 1990.

En ambos países, los acontecimientos que a partir de la década de los ochenta aceleraron el proceso de visibilización de la temática en la esfera de la opinión pública, condujeran durante la década siguiente a su consolidación en la agenda legislativa y de gobierno al mismo tiempo que ambos países adecuaban, al igual que otros de la región, sus marcos normativos en conformidad con los instrumentos internacionales introduciendo el principio de igualdad y la prohibición de discriminación de la mujer (v.gr. reconocimiento de los Tratados Internacionales de Derechos Humanos en la reforma constitucional de 1994 en Argentina y ratificación de la Convención de Belém Do Pará por México en 1995 y por Argentina en 1996) y se creaban y desarrollaban instancias o mecanismos para el tratamiento del problema (Guzmán, 2001; Sanz, 2002; Rannauro e Valdez, 2006).

En el caso de México, a fines de la década de los ochenta comienzan a parecer los primeros dispositivos estatales orientados al tratamiento de la temática. Particularmente en la Ciudad de México, se crean el Centro de Orientación y Apoyo a Personas Violadas (COAPEVI) en 1988; la primera Agencia Especializada del Ministerio Público en Delitos Sexuales (AEDS) en 1989; el Centro de Atención Integral a la Víctima de Violencia Intrafamiliar (CAVI) en 1990; y el Centro de Terapia de Apoyo a las Víctimas de Delitos Sexuales (CTA) en 1991.

Pero de particular importancia histórica es el modo en que el impulso dado por el activismo feminista a la lucha contra la violación y la violencia sexual conduce en 1991 a la reforma del Código Penal Federal en lo ateniente a los delitos sexuales. Si bien ya en 1984 se habían agravado las penas para los casos de violación, haciéndola un delito no excarcelable, y se contarán con proyecto de reforma, es en ocasión del comienzo de la presidencia de Salinas de Gortari y como resultado de estas iniciativas impulsadas por grupos feministas de diferentes sectores políticos, se produce la Reforma del Código Penal de 1991 que, entre otras cosas, eliminó expresiones sexistas como "atentados contra el pudor" y la definición de la víctima como “casta y honesta”, así 
como se redefinió el delito de violación e incorporó el hostigamiento sexual (De Barbieri, 1990).

Por su parte, durante la presidencia de Ernesto Zedillo se incluyó directamente en el Plan Nacional de Desarrollo para el sexenio presidencial 1994-2000 el objetivo de "erradicación" de la violencia contra las mujeres, la elaboración del Programa Nacional de la Mujer 1995-2000; se sancionó la Ley de Asistencia y Prevención de la Violencia Familiar para el Distrito Federal en 1997, a instancias de la cual se establecen las primeras Unidades de Atención de la Violencia Intrafamiliar (UAVIF); se lanzó el Programa Nacional contra la Violencia Intrafamiliar 1999-2000 y el Proyecto de Norma Oficial Mexicana para la Atención Médica de la Violencia Familiar en 1999 (Bedregal, 1991; Bartra, 1992; Lang, 2003; Díaz Pérez, 2009).

Para el caso de la Argentina, cabe destacar la creación de la Subsecretaría de la Mujer en 1987, y del Programa Mujer y Desarrollo en el ámbito del Ministerio de Salud y Acción Social, y de la Dirección de la Mujer en el Ministerio de Relaciones Exteriores, dependiente de la Secretaría de Derechos Humanos; así como la posterior creación del Consejo Nacional de la Mujer en 1992 (Leonor Brown, 2008).

Ahora bien, en 1988 se crea la Red Nacional contra la Violencia Doméstica, con la participación de organizaciones de todo el país, impulsada entre otras cosas por las repercusiones del caso Muñiz, que por el impacto que tuvo en la esfera pública constituyó un caso emblemático y un hito en la visibilización de la violencia de género formulada sobre todo en ese momento en términos de "violencia doméstica". El impulso dado a esta temática se refleja en la creación de la Dirección General de la Mujer del Gobierno de la Ciudad de Buenos Aires, en 1989, especialmente de las áreas de Servicio Público de Prevención y Asistencia Integral de la Violencia Familiar y de los Centros Integrales de la Mujer (CIM), dependientes de ella a partir de 1990. La violencia familiar sería, además, el primer objeto de formalización jurídica con la sanción de la Ley Nacional sobre Protección contra la Violencia Familiar (L.N. 24.417) en diciembre de 1994, si bien existían proyectos a tal fin desde mediados de los años ochenta. La atención a dicho problema daría lugar a la creación de la Oficina de Asistencia Integral a la Víctima del Delito (OFAVI), en 1998, en el marco de la Procuración General de la Nación e inicialmente orientada al abordaje de la violencia familiar (Rodríguez et al., 1997; López Oliva, 2006).

Por otro lado, en la Argentina es recién en 1999, en un momento en el que la llamada "inseguridad ciudadana" como problema social ya se había instalado definitivamente en la agenda pública y se había consolidado una demanda social de endurecimiento punitivo contra el delito en general, que se produciría la reforma del Código Penal en lo relativo a los Delitos contra la Integridad Sexual de las Personas a través de la Ley Nacional 25.087 que entre otras cosas eliminó la anacrónica figura de "delitos contra la honestidad", pero que, a diferencia del caso mexicano, no incluyó una tipificación del hostigamiento o acoso sexual (Fellini e Sansone, 2000). Dicho proceso de visibilización de la violencia de género se producía en un momento histórico complejo en el que se asistía en ambos países a procesos de democratización o consolidación de la institucionalidad democrática y a la llamada "modernización" del Estado que daría lugar a diferentes formas de articulación entre lo público y lo privado y a un incrementado protagonismo de la sociedad civil a través de diferentes formas de activismo y de mecanismos de participación ciudadana así como de intervención en la esfera de la opinión pública (Novaro, 2009; Woldenberg, 2012). Al mismo tiempo, las reformas económicas neoliberales y el desmantelamiento de los dispositivos estatales de bienestar social producían un aumento de la desigualdad y la exclusión social, a lo cual se asociaba el aumento de la violencia urbana y del miedo a la victimización en los sectores poblacionales metropolitanos (Ciafardini, 2006; Fondevila, 2007).

Esto permite reconocer que existen continuidades entre ambos casos de estudio respecto de, por un lado, el desarrollo de procesos de democratización o consolidación de la institucionalidad democrática coexistentes con transformaciones vinculadas con la liberalización comercial y la desregulación de la actividad económica y con el achicamiento del rol social del Estado; por otro, el desenvolvimiento de procesos de integración a la comunidad internacional con la adecuación de sus marcos normativos a los tratados internacionales vinculados con los Derechos Humanos, contexto en el cual en ambos países se produjo la emergencia de formas de visibilización en la esfera pública de la violencia contra la mujer, la inclusión de la temática en las instituciones de gobierno y la aparición de organizaciones no gubernamentales dedicadas a la misma.

Sin embargo, cabe ser destacado el contraste entre un pasado socio-político como el de México, caracterizado por la hegemonía del Partido Revolucionario Institucional (PRI) que gobernó durante setenta años, y la recurrencia en nuestro país de golpes de Estado cívico-militares que concluyen con el genocidio llevado adelante por el último gobierno de facto. Ello trae aparejado, entre otras cosas, que una de las aristas insoslayables respecto de la temática en la historia reciente argentina sea la consideración de la violencia de género como una práctica específica del terrorismo de Estado, lo cual ha introducido la dimensión de la memoria en sus formas de problematización durante la postdictadura (Sonderéguer e Correa, 2009; Vasallo, 2011) ${ }^{3}$. Por 
otro lado, el desarrollo en México de una llamada "guerra contra el crimen organizado" ha establecido un escenario actual de violencia social y colectiva sin parangón con la realidad presente de la Argentina (Pereyra, 2012).

Esto nos lleva al segundo período de esta genealogía, que se corresponde con la década de los dos mil y que está particularmente influenciado por las repercusiones producidas por los asesinatos sistemáticos de mujeres de Ciudad Juárez en México. Se trata de sucesos que tomaron notoriedad pública a partir del año 1993 pero que a raíz del caso conocido como "Campo Algodonero" de 2001 produjeron repercusiones no solo a nivel nacional sino internacional, ya que condujo a una sentencia pionera en materia de Derechos Humanos de las mujeres en 2009, cuando la Corte Interamericana de Derechos Humanos falló en contra del Estado mexicano. En ese proceso, ha sido destacada la labor de la Comisión Especial para Investigar los Feminicidios en México de la Cámara de Diputados de ese país, presidida entre 2003 y 2006 por Marcela Lagarde, que promovió en 2007 la Ley General de Acceso de las Mujeres a Una Vida Libre de Violencia. Esta incluyó, como una novedad particular, la definición de la "violencia feminicida" como "la forma extrema de violencia de género contra las mujeres, producto de la violación de sus derechos humanos, en los ámbitos público y privado, conformada por el conjunto de conductas misóginas que pueden conllevar impunidad social y del Estado y puede culminar en homicidio y otras formas de muerte violenta de mujeres". A lo cual, en el año 2012, se agregaría la figura de "feminicidio" como delito y se lo introduciría en el Código Penal Federal (art. 325) definido, en el contaxto normativo establecido por la Ley de 2007, como la "privación de la vida una mujer por razones de género" (Incháustegui e de la Paz, 2011). Además, a instancias de dicho caso, no solo se legitimó en el ámbito hispanoparlante el término de "feminicidio" o "femicidio", sino que se lo consolidó como figura jurídica que ha sido o está en proceso de ser incluido en los órdenes normativos de diferentes países de la región aunque de diferentes maneras que no deben ser pasadas por alto (Toledo Vásquez, 2009).

En lo que se refiere a la Argentina, en el año 2009 se establecía la "Ley de protección integral para prevenir, sancionar y erradicar la violencia contra las mujeres en los ámbitos en que desarrollen sus relaciones interpersonales", que del mismo modo que la norma mexicana, constituye una ley de segunda generación en la que, siguiendo los lineamientos de la Convención de Belem do Pará, se busca dar cuenta de manera exhaustiva de las diversas formas, modalidades y ámbitos de violencia contra las mujeres. Cabe destacar, de todos modos, que la norma argentina, si bien establece una definición de la "violencia contra la mujer" entendiéndola en base a "una relación de poder desigual" (art. 4), no incluye una referencia respecto de la violencia feminicida del modo en que lo hace la mexicana. Como quiera que sea, también en la Argentina, este recorrido ha conducido a una reforma del Código Penal en 2012 (Ley $\mathrm{N}^{\mathrm{o}}$ 26.791). Sin embargo, a diferencia de la reforma mexicana que -como mencionamos- introduce al "feminicidio" como un tipo delictivo novedoso y específico vinculado con una definición de la "violencia feminicida" que la considera en términos de una violación a los derechos humanos y llama la atención sobre los contextos de impunidad en los que se produce, en el caso argentino se realizaron una serie de modificaciones al artículo 80 que define el "homicidio agravado" agregando la dimensión de género justamente como un agravante entre otros del delito de homicidio (Buompadre, 2013).

Por otro lado, cabe destacar que la norma argentina de 2009 tiene la particularidad de incluir entre los tipos de violencia a la "violencia mediática" definida como "aquella publicación o difusión de mensajes e imágenes estereotipados a través de cualquier medio masivo de comunicación, que de manera directa o indirecta promuevan la explotación de mujeres o sus imágenes, injurie, difame, discrimine, deshonre, humille o atente contra la dignidad de las mujeres, como así también la utilización de mujeres, adolescentes y niñas en mensajes e imágenes pornográficas, que legitimen la desigualdad de trato o construyan patrones socioculturales reproductores de la desigualdad o generadores de violencia contra las mujeres" (art. 6).Y si bien la ley no lo tematiza específicamente, participa de un momento en el que las formas de problematización de la violencia de género han incorporado la temática de la trata con fines de explotación sexual, cuestión que en Argentina fue objeto de atención legislativa con la sanción de la Ley 26.364 de Prevención y Sanción de la Trata de Personas y Asistencia a su Víctimas de 2008, ley sancionada conforme al Protocolo de las Naciones Unidas para Prevenir, Reprimir y Sancionar la Trata de Personas, Especialmente Mujeres y Niños (Protocolo de Palermo), derivado de la Convención Internacional contra el Crimen Organizado Trasnacional, de 2003, perspectiva que México ya había incorporado en 2007 (Casillas, 2012; Nejamkis e Castiglione, 2013).

Finalmente, como hemos adelantado, un elemento significativo respecto de las formas de problematización de la violencia de género en el caso de Argentina está vinculado con el modo en que la emergencia de esta temática ha producido una reconfiguración de los relatos que articulan las formas de rememoración de la violencia dictatorial. Esto es el resultado también de un recorrido genealógico particular en el que ocupan un lugar fundamental las declaración de nulidad de las leyes de Punto Final y Obediencia Debida, en 2003, y de la inconstitucionalidad de los indultos concedidos por Carlos Menem, en 2006, que permitieron la reapertura de los procesos penales por los delitos de lesa humanidad cometidos durante la última dictadura cívico-militar. En este nuevo contexto estaban dadas las condiciones para 
el reconocimiento de la especificidad de la violencia de género como práctica del terrorismo de Estado, la cual había quedado invisibilizada en las primeras décadas de la posdictadura, como por ejemplo en el Juicio a las Juntas, por su inclusión en figuras jurídicas tales como las de "tormentos" y "vejaciones". Esto daría lugar a acontecimientos paradigmáticos como son el fallo dictado en junio de 2010 por el Tribunal Oral Federal de Mar del Plata que consideró por primera vez como delitos de lesa humanidad las violaciones sexuales cometidas contra mujeres en un centro clandestino de detención y exterminio (La Cueva); así como el fallo dictado por el juez federal Nro. 1 de Tucumán por el cual Luciano Benjamín Menéndez y Antonio Domingo Bussi fueron procesados como "partícipes necesarios" por la violencia de género perpetrada contra todas las mujeres detenidas en un centro clandestino de detención que funcionó en el penal de Villa Urquiza, en Tucumán, un sentencia que además llama la atención explícitamente sobre la necesidad de visibilización de la violencia de género en ese contexto (Sonderéguer, 2012).

\section{A modo de cierre}

Del mismo modo que, como hemos situado a lo largo del presente trabajo, la violencia de género en Latinoamérica posee características específicas y constituye un problema de particular importancia, también es cierto que nuestra región ha desempeñado un rol de vanguardia en lo que respecta a las luchas en pos de su visibilización que han incluido, entre otras cosas, un reconocimiento de la dimensión cultural de la conformación del problema en cuestión. De ahí se desprende la importancia de la investigación de la temática en su dimensión regional, en la cual el estudio comparado de casos permite delimitar las generalidades y las particularidades involucradas en los diferentes contextos culturales que son sometidos a indagación.

En este caso, se han presentado los aspectos fundamentales y los primeros avances de una investigación que atiende a estas consideraciones y lo hace además desde una perspectiva histórico-crítica que aborda la genealogía del problema en la historia reciente de México y Argentina.

Tal y como se ha detallado, las características del objeto a indagar, de los casos elegidos y del período de análisis configuran una investigación que posee una complejidad particular en tanto, por un lado, demanda un grado de inmersión cultural necesario para comprender las condiciones de producción y circulación de los discursos e interpretar las formas de producción de sentido en dos contextos diversos; y, por otro lado, como se ha mencionado, por el modo en que la violencia de género constituye un objeto problemático, que involucra múltiples aristas como las que hacen a la salud fisica y mental de las víctimas, al desarrollo social, a los
Derechos Humanos, etc., y cuya comprensión demanda un enfoque interdisciplinario.

Por último, no puede dejar de tenerse en cuenta que la investigación académica respecto de una temática como esta debe poder contribuir al campo de los estudios sobre la violencia de género en particular, y la violencia social en general, al mismo tiempo que colaborar a enriquecer las culturas ciudadanas y las políticas relativas al problema, a través de la producción de resultados y conclusiones que constituyan aportes de utilidad tanto para la comprensión conceptual del problema como para la conformación de modos de intervención destinados a su erradicación.

\section{Referencias}

Amnistía Internacional. La trampa del género. Mujeres, violencia y pobreza. Madrid: Amnistía Internacional, 2009.

ALMERÁS, Diane; CALDERÓN MAGAÑA, Coral (Eds.). Si no se cuenta, no cuenta. Información sobre la violencia contra las mujeres. Santiago de Chile: ONU/CEPAL, 2012.

AUSTIN, John. Cómo hacer cosas con palabras. Buenos Aires: Paidós, 2003.

BARRANCOS, Dora. Mujeres. Entre la casa y la plaza. Buenos Aires: Sudamericana, 2012.

BARTRA, Eli. Mujeres y política en México: aborto, violación y mujeres golpeadas. Política y Cultura, n. 1, UAM, 1992.

BEDREGAL, Ximena (Ed.). Tres experiencias en la Ciudad de México. México: CICAM, 1991.

BHASIN, Kamla. Mujeres y las alternativas de comunicación. Bangkok: Encuentro Mundial "La Comunicación como fuente de poder para las mujeres”, 1994.

BOLLES, Augusta Lynn; LEBON, Nathalie; MAIER, Elizabeth. De lo privado a lo público: 30 años de lucha ciudadana de las mujeres en América Latina. México: UNIFEM / Siglo XXI, 2006. BONNET, Alberto. La hegemonía menemista. El neoconservadurismo en Argentina, 1989-2001. Buenos Aires: Prometeo, 2007.

BORRAT, Héctor. El periódico, actor político. Barcelona: Gustavo Gili, 1989.

BRENDEL, Christine. La lucha contra la trata de mujeres en Centro América y El Caribe. Eschborn: GTZ 2003.

BUOMPADRE, Jorge Eduardo. Los delitos de género en la reforma penal (Ley No 26.791). Revista Pensamiento Penal, $\mathrm{n}$. 152, 2013.

CADENA VARGAS, Edel. El neoliberalismo en México: saldos económicos y sociales. Quivera, n. 7, UAEMEX, 2005. CARRILLO, Roxana; BUNCH, Charlotte. Gender Violence: a development and Human Rights issue. New Jersey: Center for Women's Global Leadership, 1991.

CASILLAS, Rodolfo. La mundialización del delito. Redes de tráfico y trata de personas en México. Nueva Sociedad, n. 241, 2012.

CECYM. Feminismo por feministas. Fragmentos para una 
historia del feminismo argentino 1970-1996. Travesías 5. Buenos aires: CECYM, 1996.

CHEJTER, Silvia. Adriana Mabel Montoya 1983 Buenos Aires. Violación: discurso jurídico y discurso de la prensa escrita. Buenos Aires: CECYM, 1995.

CIAFARDINI, Mariano. Delito urbano en la Argentina. Buenos Aires: Ariel, 2006

CONTRERAS, Juan Manuel et al. Violencia sexual en Latinoamérica y El Caribe: análisis de datos secundarios. Washington: ICRW / OPS, 2010.

DE BARBIERI, Teresita. Ni tanto ni tan poco : las reformas penales relativas a la violencia sexual. Debate Feminista, año I vol. 2, 9/1990, v. I, n. 2, 1990.

DE MATTOS, Carlos. Globalización y metamorfosis urbana en América Latina. Quito: OLACCHI, 2010.

DEAN, Mitchell. Critical and effective histories: Foucault's methods and historical sociology. Routledge, 1994.

DÍAZ BARRADO, Mario (Ed.). Historia del tiempo presente: Teoría y metodología. Cáceres: Universidad de Extremadura, 1998.

DÍAZ PÉREZ, Guillermina. La violencia de género en México: reto del gobierno y de la sociedad. Encrucijada, n. 2, UNAM, 2009.

DREZIN, Jenny (Ed.). Imaginemos una vida sin violencia: estrategias de comunicación y de los medios para terminar con la violencia contra las mujeres. New York: UNIFEM, 2001. FALÚ, Ana (Ed.). Mujeres en la ciudad. De violencias y derechos. Santiago de Chile: SUR, 2009.

FALÚ, Ana; RAINERO, Liliana (Eds.). Hábitat urbano: una visión de género. Córdoba: CICSA-SAREC, 1995.

FELLINI, Zulita; SANSONE,Virginia. La mujer en el derecho penal argentino Anuario de Derecho Penal, n. nro. 19992000, 2000.

FONDEVILA, Gustavo. Instituciones, legalidad y Estado de Derecho en el México de la transición democrática. México: Fontamara, 2007. FOUCAULT, Michel. La verdad y las formas jurídicas. Buenos Aires: Gedisa, 2007.

El orden del discurso. Buenos Aires: Tusquets, 2008.

FRÍES, Lorena; HURTADO, Victoria. Estudio de la información sobre la violencia contra la mujer en América Latina y el Caribe. Santiago de Chile: ONU/CEPAL, 2010.

GAMBA, Susana. Estudios de Género/Perspectiva de Género. In: GAMBA, S. (Ed.). Diccionario de estudios de género $y$ feminismos. Buenos Aires: Biblos, 2007.

GIDDENS, Anthony. Las nuevas reglas del método sociológico: crítica positiva de las sociologías interpretativas. Buenos Aires: Amorrortu, 2001.

GIL LOZANO, Fernanda. Feminismos en la Argentina de los '70 y los '80. Buenos Aires: Instituto Interdisciplinario de Estudios de Género, UBA. 2004.

GUERRERO, Elisabeth. Informe sobre violencia contra las mujeres en América Latina y el Caribe, 1990 - 2000. Balance de una década. Santiago de Chile: ISIS Internacional/UNIFEM, 2002. GUZMÁN,Virginia. La institucionalidad del género en el estado: nuevas perspectivas de análisis. Santiago de Chile: CEPAL, 2001. HABERMAS, Jürgen. Teoría de la acción comunicativa: racionalidad de la acción y racionalización social. Madrid:Taurus, 1999.
Factibilidad y validez: sobre el derecho y el Estado democrático de derecho en términos de teoría del discurso. Madrid:Trotta, 2001.

HEISE, Lori; ELLSBER G, Mary; GOTTEMOELLER, Megan. Ending Violence Against Women. Baltimore: Johns Hopkins University, 1999.

INCHÁUSTEGUI,Teresa; DE LA PAZ, María (Eds.). Feminicidio en México. Aproximación, tendencias y cambios, 1985-2009. México: ONU Mujeres / INMujeres / Comisión Especial para el Seguimiento de los Feminicidios - Cámara de Diputados, 2011.

JIMÉNEZ, Patricia; RODEROS, Katherie (Eds.). Feminicidio: un fenómeno global de Lima a Madrid. Bruselas: HBS/ UE, 2010.

LACLAU, Ernesto; MOUFFE, Chantal. Hegemonía y estrategia socialista. Hacia una radicalización de la democracia. Madrid: Siglo XXI, 1987.

LAMAS, Marta (Ed.). El género. La construcción cultural de la diferencia sexual. México: Purrúa, 2000.

LANG, Miriam. ¿Todo el poder? Políticas públicas, violencia de género y feminismo en México. Iberoamericana, v. III, n. 12, 2003.

LEONOR BROWN, Josefina. De la institucionalización de los asuntos de las mujeres en el Estado Argentino y algunos de los avatares, entre los ochenta y los noventa. Revista Mora, v. 14, n. 2, Buenos Aires, 2008.

LÓPEZ OLIVA, Mabel.Violencia familiar en la Ciudad de Buenos Aires: Un estudio sobre la dinámica de relación entre organizaciones no gubernamentales, poder judicial y otros servicios estatales frente a las denuncias judiciales. Cuadernos de CLASPO-Argentina, n. 5, 2006.

LÓPEZ PONS, María Magdalena. La violencia de género en el territorio latinoamericano, a través de la ocurrencia creciente de los feminicidios en la región. Revista Latino-americana de Geografia e Gênero, v. 1, n. 1, 2010.

LOSEKE, Denise ; BEST, Joel (Eds.). Social Problems: Constructionist Readings. New York: Aldine De Gruyter, 2003.

MAFFIA, DIana. El feminismo y la política: retos presentes y futuros". In: (Ed.). Liderazgos políticos con perspectiva de género. Rosario: FES, 2002.

MAHONEY, James; RUESCHEMEYER, Dietrich. Comparative Historical Analysis in the Social Sciences. Nueva York: Cambridge University Press, 2003.

MAR GULIS, Mario. Sociología de la cultura: conceptos y problemas. Buenos Aires: Biblos, 2009.

MARTÍN BARBERO, Jesús. Lo público: experiencia urbana y metáfora ciudadana. CIC. Cuadernos de Información $y$ Comunicación, v. 13, n. UCM, Madrid, 2008.

MCCOMBS, Maxwell. Estableciendo la agenda: el impacto de los medios en la opinión pública y en el conocimiento. Barcelona: Paidós, 2006.

MILOSAVLJEVIC,Vivian. Estadísticas para la equidad de género: magnitudes y tendencias en América Latina. Santiago de Chile: UN/CEPAL, 2007.

MORELLI, Mariana; REY, Patricia. La violencia tiene prensa. Análisis de la cobertura periodística de la violencia contra las mujeres en los medios gráficos de América Latina. Informe 
final del Observatorio Regional "Las mujeres en los medios". Buenos Aires: ELA, 2012.

NAVARRO, Marysa. Dilemas y desafios: el primer Encuentro Feminista Latinoamericano y del Caribe, vol. III. In: LEÓN, M. (Ed.). Sociedad, subordinación y feminismo. Debate sobre la mujer en América Latina y El Caribe. Bogotá:ACEP, 1982.

NEJAMKIS, Lucila ; CASTIGLIONE, Celeste. Apuntes críticos a la trata de personas como problema contemporáneo. Revista Población y Desarrollo, v. 9, 2013.

NOVARO, Marcos. Argentina en el fin de siglo. Democracia, mercado y nación (1983-2001). Buenos Aires: Paidós, 2009.

OEA. Convención Interamericana para prevenir, sancionar y erradicar la violencia contra la mujer: Convención de Belém Do Pará. OEA, 1994.

OEA/CIDH. Acceso a la justicia para las mujeres víctimas de la violencia en las Américas. Washington: OEA - Comisión Interamericana de Derechos Humanos, 2007.

OPS/OMS. Violencia contra las mujeres. Políticas públicas sobre violencia doméstica. Washington: OPS/OMS, 2005.

PEREYRA, Guillermo. México: violencia criminal y "guerra contra el narcotráfico". Revista Méxicana de Sociología, n. 3, UNAM-IIS, 2012.

POPPE, Patricia. Medios de comunicación como colaboradores en la prevención de la violencia doméstica. Washington: The Johns Hopkins University / BID, 1997.

PORTES, Alejandro; ROBERTS, Bryan; GRIMSON, Alejandro (Eds.). Ciudades latinoamericanas: un análisis comparativo en el umbral del nuevo siglo. Buenos Aires: Prometeo, 2008. RABEY, Mario; MARTÍNEZ, José Manuel (Eds.). La Constitución de la Ciudad de Buenos Aires 1996-2006. Buenos Aires: Instituto de Políticas Públicas, 2006.

RADFORD, Jane; RUSSELL, Diane. Femicide: the politics of woman killing. New York: Twayne, 1992.

RANNAURO, Elizardo;VALDEZ, Miriam (Eds.). La eliminación de la violencia contra las mujeres en México: enfoque desde el ámbito internacional. México: SRE/UNIFEM/PNUD, 2006. RESTREPO, Alejandra; BUSTAMANTE, Ximena. 10 encuentros feministas latinoamericanos y del Caribe. Apuntes para una historia en movimiento. México: Comité Impulsor del XI Encuentro Feminista, 2009.

RICO, Nieves. Violencia doméstica contra la mujer en América Latina y el Caribe: propuestas para la discusión. Santiago de Chile: UN/CEPAL, 1992.

Violencia de género: un problema de Derechos $\mathrm{Hu}-$ manos. Santiago de Chile: CEPAL, 1996.
RODIGOU, Maite (Ed.). La violencia hacia las mujeres en los medios de comunicación. Transformando las noticias. Córdoba: CICSA, 2007.

RODRÍGUEZ, Marcela; STAUBLI, Diana; GÓMEZ, Patricia (Eds.). Mujeres en los '90. Legislación y políticas públicas. Buenos Aires: Centro Municipal de la Mujer de Vicente López, 1997.

RUSSELL, Diane. The politics of rape: the victim's perspective. New York: Stein and Day, 1975.

RUSSELL, Diane; VAN DE VEN, Nicole (Eds.). Crimes Against Women: Proceedings of the International Tribunal. Berkeley: Russell Publications, 1990.

SAGOT, Montserrat. Los límites de las reformas: violencia contra las mujeres y políticas públicas en América Latina. Revista de Ciencias Sociales de la Universidad de Costa Rica, n. 120, 2008.

SANZ, Susana. La Mujer y la violencia en la República Argentina. Convenciones internacionales, legislación nacional y provincial, desafíos. Buenos Aires: Consejo Nacional de la Mujer, 2002.

SEGATO, Rita. Las estructuras elementales de la violencia. Buenos Aires: Prometeo, 2010.

SERRANO SALAZAR, Oziel. La reforma política del Distrito Federal. México: CENAM / Plaza y Valdés, 2001.

SONDERÉGUER, María. Género y poder: violencias de género en contextos de represión política y conflictos armados. Bernal: UNQ, 2012.

SONDERÉGUER, María; CORREA,Violeta. Análisis de la relación entre violencia sexual, tortura y violación a los Derechos Humanos. Bernal: UNQ, 2009.

TOLEDO VÁSQUEZ, Patsilí Feminicidio. México: Oficina en México del Alto Comisionado de las Naciones Unidas para los Derechos Humanos, 2009.

UN. Poner fin a la violencia contra la mujer: de las palabras los hechos. Nueva York: UN, 2006.

. La ONU y la mujer: compilación de mandatos. Buenos Aires: Centro de Información para Argentina y Uruguay, 2010. UNESCO. Women and the media: acces to expression and decision-making. Report of the Internacional Symposium. Toronto: UNESCO, 1995.

VASALLO, Marta (Ed.). Grietas en el silencio. Una investigación sobre la violencia sexual en el marco del terrorismo de Estado. Rosario CLADEM, 2011.

VELZEBOER, Marijke. La violencia contra las mujeres: responde el sector de la salud. Washington: OPS, 2003.

VERÓN, Eliseo. La semiosis social: Fragmentos de una teoría de la discursividad. Barcelona: Gedisa, 2004.

WOLDENBERG, José La transición democrática en México. México: COLMEX, 2012. 


\title{
Fundamentals for the comparative study of the forms of construction of gender violence as a public-political problem in Mexico and Argentina.
}

\begin{abstract}
Gender-based violence is a global problem and has special incidence in Latin America, which is why is recognized as a matter that concerns to public health, as an obstacle to development and as a serious human rights issue. This paper presents the fundamentals for the comparative study of its construction as a public-political problem in Mexican and Argentinian recent history. It also specifies the preliminary results of the comparative research on the socio-historical processes that -in both countries with its peculiarities- produced the emergency of the problem in the public sphere of the hand of feminist activism and the emergence of civil society organizations; and the incorporation into the parliamentary and governmental agendas in the context of its theming by international organizations.
\end{abstract}

Key words: gender-based violence; Latin America; Argentina; México; recent history

\section{Fundamentos para o estudo comparativo das formas de construção da violência de gênero como um problema público-político na história recente do México e da Argentina.}

\begin{abstract}
Resumo
A violência de gênero é um problema global de particular impacto na América Latina, e é por isso que foi reconhecido como um problema para a saúde, um obstáculo ao desenvolvimento e uma questão séria de direitos humanos. Este trabalho dá conta dos fundamentos para o estudo comparativo de sua construção como problema público-político na história recente do México e da Argentina. Além disso, os resultados parciais da pesquisa comparativa dos processos sócio-históricos em ambos países, com suas peculiaridades, permite comprender as formas de emergência do problema na esfera pública nas mãos do ativismo feminista e da aparição de organizações da sociedade civil; e as formas em que o tema é incorporado nas agendas legislativas e de Governo, no contexto de sua tematização por organizações internacionais.
\end{abstract}

Palavras-chave: violência de gênero; América Latina; Argentina; México; história recente

Data de recebimento: 22-09-2015

Data de aceitação: 22-10-2015 